\title{
Influencia de la Materia Seca como Índice de Madurez de Cosecha y Tiempo de Almacenamiento en Frío sobre la Calidad del Aguacate cv. Hass Producido en la Región del Trópico Alto
}

\author{
Johanna V. Escobar(1), Pablo Rodriguez ${ }^{(2)}$, Misael Cortes ${ }^{(1) \star}$ y Guillermo Correa(1) \\ (1) Facultad de Ciencias Agrarias. Universidad Nacional de Colombia Sede Medellín, Colombia. \\ (e-mail: jovescobarca@unal.edu.co; mcortesro@unal.edu.co; gcorrea@unal.edu.co). \\ (2) Corporación Colombiana de Investigación Agropecuaria (AGROSAVIA), Colombia. (e-mail: prodrguezf@agrosavia.co)
}

*Autor a quien debe ser dirigida la correspondencia

Recibido Jul. 19, 2018; Aceptado Oct. 1, 2018; Versión final Nov. 27, 2018, Publicado Jun. 2019

\begin{abstract}
Resumen
El objetivo de esta investigación fue evaluar el efecto de la materia seca como índice de madurez de cosecha y el tiempo de almacenamiento sobre parámetros de calidad del aguacate. En cuatro fincas se cosecharon frutos a tres valores de materia seca: 22, 26 y $30 \%$. Después de la cosecha se midieron defectos externos, peso y color de los frutos. Los aguacates fueron desinfectados y almacenados $\left(5^{\circ} \mathrm{C} / 90 \% \mathrm{HR}\right.$ durante $0,3,4 \mathrm{y}$ 5 semanas). Luego del almacenamiento, se evaluaron los daños por frío y pérdida de peso. Posteriormente, los frutos se maduraron $\left(20^{\circ} \mathrm{C} / 90 \% \mathrm{HR}\right)$ y se determinaron aspectos de calidad en madurez de consumo: color y daños de la pulpa. A un valor de materia seca de $26 \pm 2 \%$, la fruta tuvo el menor nivel de defectos de pulpa para las regiones de estudio. Esto significa que, al controlar el momento de recolección del fruto y el tiempo de almacenamiento, se puede mejorar la calidad postcosecha del aguacate.
\end{abstract}

Palabras clave: aguacate; calidad; postcosecha; daños de pulpa; ; cv. Hass

\section{Influence of Dry Matter as a Harvest Index and Cold Storage Time on cv. Hass Avocado Quality Produced in High Tropic Region}

\begin{abstract}
The aim of this research was to evaluate the effect of dry matter as a harvest index and the storage time on the Hass avocado postharvest quality parameters. From four locations, fruit were harvested at three dry matter values: 22, 26 and $30 \%$. After harvest, the external defects, weight, and color fruit were analyzed. The avocados were disinfected and stored $\left(5^{\circ} \mathrm{C} / 90 \% \mathrm{RH}\right.$ during $0,3,4$ y 5 weeks). After storage, the chilling damage and weight losses were evaluated. After that, fruit were ripened $\left(20^{\circ} \mathrm{C} / 90 \% \mathrm{RH}\right)$ and the quality aspects of "ready to eat" Hass avocado: color and flesh discoloration were determined. At $26 \pm 2 \%$ of dry matter, the fruit had the lowest level of defects for the study regions. This means that the avocado fruit quality could improve if the harvest moment and storage time are controlled.
\end{abstract}




\section{INTRODUCCIÓN}

El aguacate (Persea americana Mill cv. 'Hass') es un fruto apetecido a nivel mundial por las características funcionales y sensoriales de su pulpa. El fruto contiene vitaminas, grasas poliinsaturadas, minerales y componentes activos, los cuales lo convierten en un alimento funcional (Dreher y Davenport, 2013). El aguacate se ha convertido en un fruto de importancia para el sector agrícola y económico de Colombia. Este país en los últimos años, ha incrementado sus exportaciones principalmente a Europa durante el periodo del 2010 al 2016 (52,7 a $17829 \mathrm{Mg}$ ) y con unas perspectivas de mayor expansión al mercado internacional (Agronet, 2017), en especial, con la aprobación por parte del APHIS del ingreso al mercado de Estados Unidos en agosto de 2017. En Colombia, el cultivo del aguacate 'Hass' inició en el año 2000 y desde entonces, ha tenido un importante ascenso productivo y económico, lo que ha generado la necesidad de evaluar parámetros de calidad no solo a nivel de cultivo, sino también en la postcosecha, con el fin de ofrecer conocimiento y tecnologías para fortalecer la competitividad de la cadena. El fruto de aguacate no madura en el árbol, por lo que se debe recurrir a un periodo de maduración (con temperatura superior a $17^{\circ} \mathrm{C}$ ) antes del consumo (Arpaia et al., 2015). Sin embargo, los países productores de aguacate (Chile, Sudáfrica, Colombia, entre otros), están distantes de los mercados principales y para enviar el producto utilizan técnicas de conservación como la refrigeración y las atmosferas controladas (Zauberman y Jobin, 1995; Ochoa, 2014). Una vez que el fruto se somete a maduración (etapa que se realiza en el mercado de destino), se han observado a nivel internacional problemas fisiológicos y de calidad en la postcosecha del aguacate. Por tanto, la investigación sobre esta problemática en los países productores y consumidores, es de alta importancia (Pedreschi et al., 2014; Hernández et al., 2015). Algunos de estos desórdenes o defectos postcosecha son provocados por el estrés por frío, traducido en: pérdidas de peso, deshidratación, defectos superficiales en la epidermis del fruto, heterogeneidad en la maduración o "tablero de ajedrez", pardeamiento y oscurecimiento de la pulpa. Estos desordenes provocan problemas logísticos y de aceptación de los frutos por los consumidores (Ferreyra y Defilippi, 2012).

Para alcanzar adecuados atributos de calidad y minimizar los defectos de la postcosecha, es usado el índice de madurez de cosecha, como un indicador de calidad y a su vez un parámetro de decisión en la recolección de los frutos. El indicador más empleado internacionalmente en esta especie es el contenido de materia seca de la pulpa del fruto. Sin embargo, para cada país productor la acumulación de materia seca, depende de las condiciones ambientales, de manejo de cultivo y suelos, por ejemplo, en USA y Australia, los frutos son recolectados a partir de 20,6\% de materia seca (United-Nations Ffv-42, 2010), mientras que en Costa Rica para climas intermedios se cosechan a $23 \%$ (Cerdas et al., 2014). En México, la cercanía a su principal mercado de destino conlleva a que los frutos puedan ser cosechados con materia seca superior al $30 \%$. En Colombia la mayoría de los frutos de aguacate Hass son cultivados por encima de 2000 m.s.n.m (Bernal et al., 2014) y no se tiene información sobre indicadores de calidad e impacto en la vida útil de los frutos al llegar al mercado de destino. Por estas razones, el objetivo de esta investigación fue evaluar la influencia del índice de madurez de cosecha por materia seca y el tiempo de almacenamiento en frío bajo condiciones simuladas de exportación, sobre la calidad del aguacate 'Hass', en diferentes zonas de cultivo de Antioquia (Colombia).

\section{MATERIALES Y MÉTODOS}

Las muestras de aguacate Hass se obtuvieron de cuatro lotes de fincas exportadoras (con certificación Global GAP) de sub-regiones de Antioquia (Norte, Oriente, Sur-Oeste). En cada finca se marcaron 400 frutos al azar, en 30 árboles previamente seleccionados (de 7 a 10 años de edad) entre el año 2015 y 2016. De manera periódica (cada 15 días) y durante el crecimiento de los frutos se realizaron muestreos aleatorios, con el fin monitorear el contenido de materia seca y cosechar los frutos en tres índices de madurez (IM): 22, 26 y 30\%.

Tabla 1: Caracterización de las fincas utilizadas en el estudio, con relación a la edad de los árboles, su posición geográfica, altitudinal y la temperatura promedio de la zona

\begin{tabular}{|l|c|c|c|c|}
\hline \multicolumn{1}{|c|}{ Fincas } & $B V($ Sur-Oeste) & $E B$ (Norte) & LE (Oriente) & CS (Norte) \\
\hline Edad árboles (años) & 10 & 8 & $8-9$ & $8-9$ \\
\hline Latitud & $05^{\circ} 35^{\prime} 50,2^{\prime \prime}$ & $6^{\circ} 29^{\prime} 39,6^{\prime \prime}$ & $6^{\circ} 05^{\prime} 57,0^{\prime \prime}$ & $6^{\circ} 29^{\prime} 23,8^{\prime \prime}$ \\
\hline Longitud & $075^{\circ} 48^{\prime} 19,8^{\prime \prime}$ & $75^{\circ} 31^{\prime} 42,0^{\prime \prime}$ & $75^{\circ} 26^{\prime} 14,9^{\prime \prime}$ & $75^{\circ} 31^{\prime} 29,1^{\prime \prime}$ \\
\hline $\mathrm{msnm}$ & 2025 & 2464 & 2167 & 2411 \\
\hline $\mathrm{T}\left({ }^{\circ} \mathrm{C}\right)$ anual promedio & 19,0 & 16,0 & 15,7 & 15,8 \\
\hline
\end{tabular}

Una vez los aguacates alcanzaron los valores predeterminamos de materia seca, se recolectaron 50 frutos por IM y por cada finca. Las muestras se transportaron al centro de investigación La Selva de AGROSAVIA, (Rionegro-Antioquia). Se tomó una sub muestra al azar (correspondiente al $10 \%$ del total de los frutos) para determinar la materia seca (Tabla 2) en el momento de cosecha, siguiendo el método 934.06 AOAC (2000) 
con algunas modificaciones, como el peso de la muestra $(5 \mathrm{~g})$ y el tiempo de secado (5 horas) de los frutos hasta obtener un peso constante.

Tabla 2: Porcentaje de materia seca determinada en 5 frutos de aguacate 'Hass' individuales por finca y fecha de cosecha.

\begin{tabular}{|l|l|l|}
\hline \multicolumn{1}{|c|}{ Finca/subregión } & \multicolumn{1}{|c|}{ Fecha de cosecha } & Promedio de materia seca (\%) \\
\hline CS (Norte) & $14-j u n-2016$ & $22,09 \pm 0,99$ \\
\hline CS (Norte) & 26 -julio-2016 & $25,44 \pm 2,08$ \\
\hline CS (Norte) & 09 -agos-2016 & $29,97 \pm 0,44$ \\
\hline BV (sur-oeste) & $07-$ oct-2016 & $22,04 \pm 1,07$ \\
\hline BV (sur-oeste) & 11 -nov-2016 & $26,24 \pm 1,18$ \\
\hline BV (sur-oeste) & 18 -ene-2017 & $29,48 \pm 0,68$ \\
\hline EB (Norte) & 29 -nov-2017 & $22,58 \pm 2,04$ \\
\hline EB (Norte) & 28 -dic-2016 & $26,48 \pm 2,08$ \\
\hline EB (Norte) & $25-01-2016$ & $30,73 \pm 1,54$ \\
\hline LE (oriente) & 30 -dic-2017 & $21,73 \pm 1,94$ \\
\hline LE (oriente) & 10 -ene-2017 & $24,49 \pm 1,75$ \\
\hline LE (oriente) & $03-$ feb-2017 & $29,51 \pm 1,41$ \\
\hline
\end{tabular}

Calidad de frutos de aguacate, medidos después de la cosecha

Los daños externos fueron analizados siguiendo lo establecido en el manual internacional de la calidad de aguacate de White et al., (2009), en el cual se indica la escala de severidad de daño de 0 a 3 (siendo 0 : ausencia de daño, $0,5: 5 \%, 1: 10 \%, 1,5: 15 \%, 2: 25 \%, 2,5: 33 \%$ y $3 \geq 50 \%$ de la superficie del fruto presenta el daño). Los daños evaluados al momento de la recepción de frutos fueron los daños provocados por roce, daño mecánico, golpes de sol y daños por insectos, específicamente por el coleóptero marceño (Astaena sp,) y trips, en frutos clasificados como de exportación. El 90\% de los frutos que quedaron para cada tratamiento fueron codificados y pesados (peso inicial) uno a uno con una balanza de precisión de $0,01 \mathrm{~g}$ (Meter PE 3600). El color de la muestra en madurez de cosecha y de consumo fue medido con un colorímetro portátil (Minolta modelo CR410, iluminante $C$ y observador de $2^{\circ}$ ). Los resultados se expresan en la escala CIE $L^{*} a^{*} b^{*}$ y ángulo de tono $h^{\circ}$ (hue). Cada medida fue realizada en el epicarpio del fruto, sobre la zona ecuatorial, donde se observó uniformidad en el color a simple vista y delimitada con un marcador sharpie-color dorado (Cox et al., 2004).

\section{Condiciones de almacenamiento de los frutos}

La desinfección de los frutos se hizo con un fungicida comercial (prochloraz al 0,05\% p/v, Mirage® $45 \mathrm{EC}$,), luego secado al ambiente y empacado en cajas comerciales de cartón corrugado. Posteriormente, el almacenamiento se realizó en cámara frigorífica (Memmert HPP 110) con una temperatura y humedad relativa de $5 \pm 0,1^{\circ} \mathrm{C}$ y $90 \pm 0,5 \% \mathrm{HR}$, respectivamente. Se utilizaron tres periodos almacenamiento (TA) de 3,4 y 5 semanas. Al finalizar cada periodo de almacenamiento, se tomaron 8 frutos para cada tratamiento (anteriormente codificados) y se pesaron en una balanza (meter PE 3600, precisión de 0,01g). Las diferencias de peso fueron reportadas como el porcentaje de pérdida de peso en almacenamiento (PA). También se evaluaron los daños por frío sobre la epidermis de los frutos, siguiendo la metodología propuesta por White et al., (2009).

Posteriormente, los frutos fueron situados para su maduración en una cámara Memmert HPP 110 a $20,0 \pm 0,1^{\circ} \mathrm{C}$ y $90,0 \pm 0,5 \% \mathrm{HR}$ (Burdon et al., 2013; Bill et al., 2014; Arpaia et al., 2015). La muestra control que no fue sometida a refrigeración, se maduró en cámara inmediatamente después de su acondicionamiento. Periódicamente, se realizó inspección a la muestra, para suspender la maduración cuando los frutos llegaron al grado 5 de madurez: color $75 \%$ purpura de la epidermis y firmeza al tacto suave (índice bajo $1 \mathrm{~mm}$ de deformación en la zona ecuatorial con personal previamente entrenado) que corresponde a 2,0 $\mathrm{Kgf}_{\mathrm{cm}}^{-2}$ aproximadamente, medidos en la zona ecuatorial del fruto hasta que se rompía la epidermis y llegará a la pulpa del aguacate sin tocar la semilla (White et al., 2009; Zapata et al., 2016). Se registraron los días a madurez de consumo y el porcentaje de pérdida de peso (PM) de los frutos para cada tratamiento.

Medición de la calidad en frutos en estado de madurez de consumo

El área afectada $(\mathrm{AA})$ por daños en la pulpa de los frutos, se determinó con un software de análisis de imagen (desarrollado en el Cl La Selva de Agrosavia). Este dispositivo, está basado en principios de visión 
computacional, en el cual se controla la iluminación, la ubicación de la muestra y se captura las imágenes con la cámara web superior. Luego el dispositivo es conectado al software "Persea" Python, openCV (Figura 1) realizando dos segmentaciones, la primera para extraer el contorno del aguacate y la segunda para identificar el daño ubicado dentro de dicho contorno. El dispositivo "Persea" automáticamente calcula el área de la imagen que corresponde al daño como porcentaje de superficie afectada.

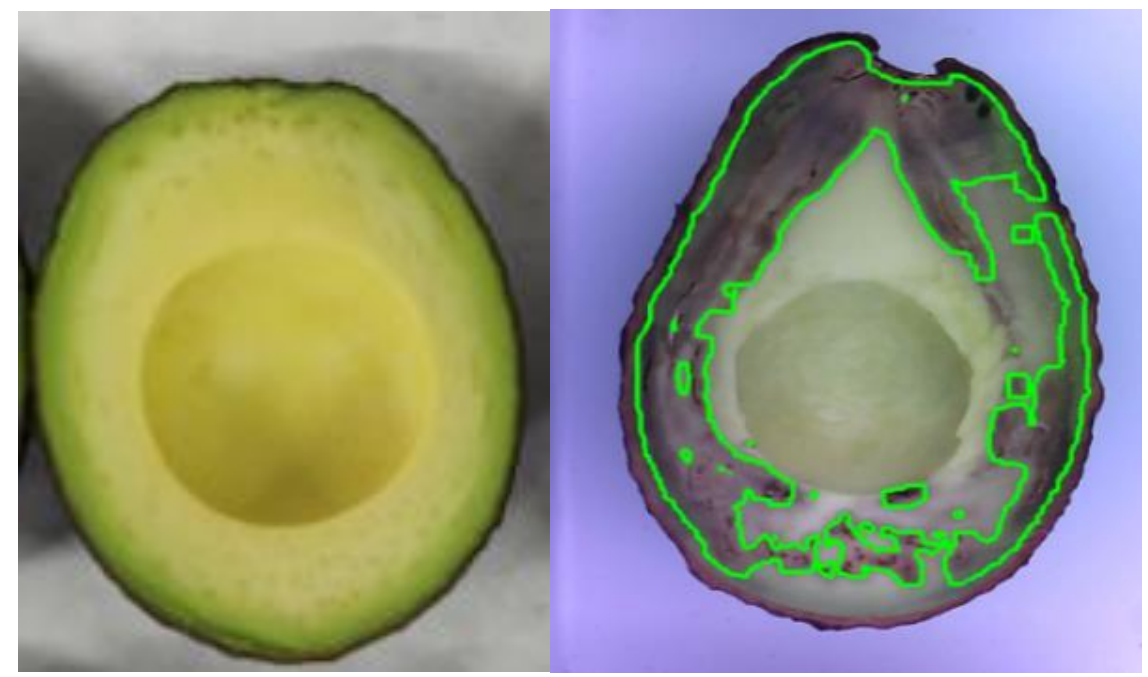

Fig. 1: Determinación cuantitativa del daño en la pulpa de los frutos mediante el uso de un software de análisis de imagen ("Persea" (Software Python, openCV).

\section{Análisis experimental y estadístico}

Mediante un ANOVA de dos vías con un nivel de confianza del 95\%, se midió los Índices de madurez (22, 26 y $30 \%$ ) y el tiempo de almacenamiento en frío $(0,3,4,5$ semanas) para cada finca seleccionada (BV, EB, CS, LE) con su interacción. Los resultados de las secciones de análisis: después de la cosecha, almacenamiento y maduración que cumplieron supuestos de normalidad, se expresaron a través de su media aritmética y la desviación estándar. Para determinar si existían diferencias entre los tratamientos, se realizó la comparación de las medias, mediante la prueba de Tukey HSD con un nivel de confianza del $95 \%$.

Para los daños externos se realizó una prueba de Dunn de comparación de medias con nivel de confianza del $95 \%$. Finalmente, se ajustó una superficie de respuesta para el área afectada (AA). Los análisis fueron realizados en el software estadístico Statgraphics Centurión versión XVI. y el paquete estadístico R (R Core Team, 2017).

\section{RESULTADOS Y DISCUSIÓN}

Los resultados son presentados en tres secciones: Calidad de frutos de aguacate, medidos después de la cosecha, parámetros de calidad medidos después del almacenamiento en frío y parámetros de calidad en aguacate "listo para comer".

\section{Calidad de frutos de aguacate, medidos después de la cosecha}

Los daños externos son aquellos ocasionados durante la pre cosecha, cosecha y empacado de los frutos de aguacate. Estos daños son usados como parámetros de selección y se establecen como criterios de categorización de la calidad de acuerdo a la normatividad vigente en cada país (NMX, 2006, 1994; NTE, 2009; OECD, 2004). En la figura 2 se observa que el daño por roce o lenticelosis es el mayor daño externo encontrado para las cuatro fincas estudiadas (BV, EB, CS y LE), se tuvieron valores de daño entre el 60 y $95 \%$. Aunque la severidad en cada fruto estuvo entre 5 al $10 \%$, la lenticelosis puede promover daños por frío, pérdida de humedad o inducir a la maduración prematura debido a la muerte celular en la zona del epicarpio del fruto (Ochoa, 2014).

El daño por insecto (trips) se encontró entre el 40 y $50 \%$, sin presentar diferencias estadísticamente significativas $(P<0,05)$ para las fincas EB y BV. Las fincas CS y LE tuvieron valores inferiores al $25 \%$. El ataque por marceño (Astaena $s p$,) tuvo una frecuencia menor al $20 \%$ del total de los frutos. Los daños causados por los dos insectos (trips y marceño) son permitidos a bajos valores de severidad, para exportación, por considerarse daños estéticos (encrestamientos o venas y costras en la piel que no causan daño en pulpa), a pesar de ello, los frutos pueden ser rechazados o poco valorados en el mercado 
internacional por su apariencia y reducen su valor en el mercado (Bernal et al., 2014). Los daños mecánicos (como cortaduras superficiales), al igual que los daños causados por golpes de sol, se encontraron en menores proporciones $(<33 \%)$. Finalmente, se puede afirmar que la mayoría de frutos evaluados se encuentran en categoría Extra o categoría I, de acuerdo con la normatividad internacional (OECD, 2004), lo cual sugiere que el proceso de selección en campo permite cumplir la normatividad internacional vigente para este fruto.
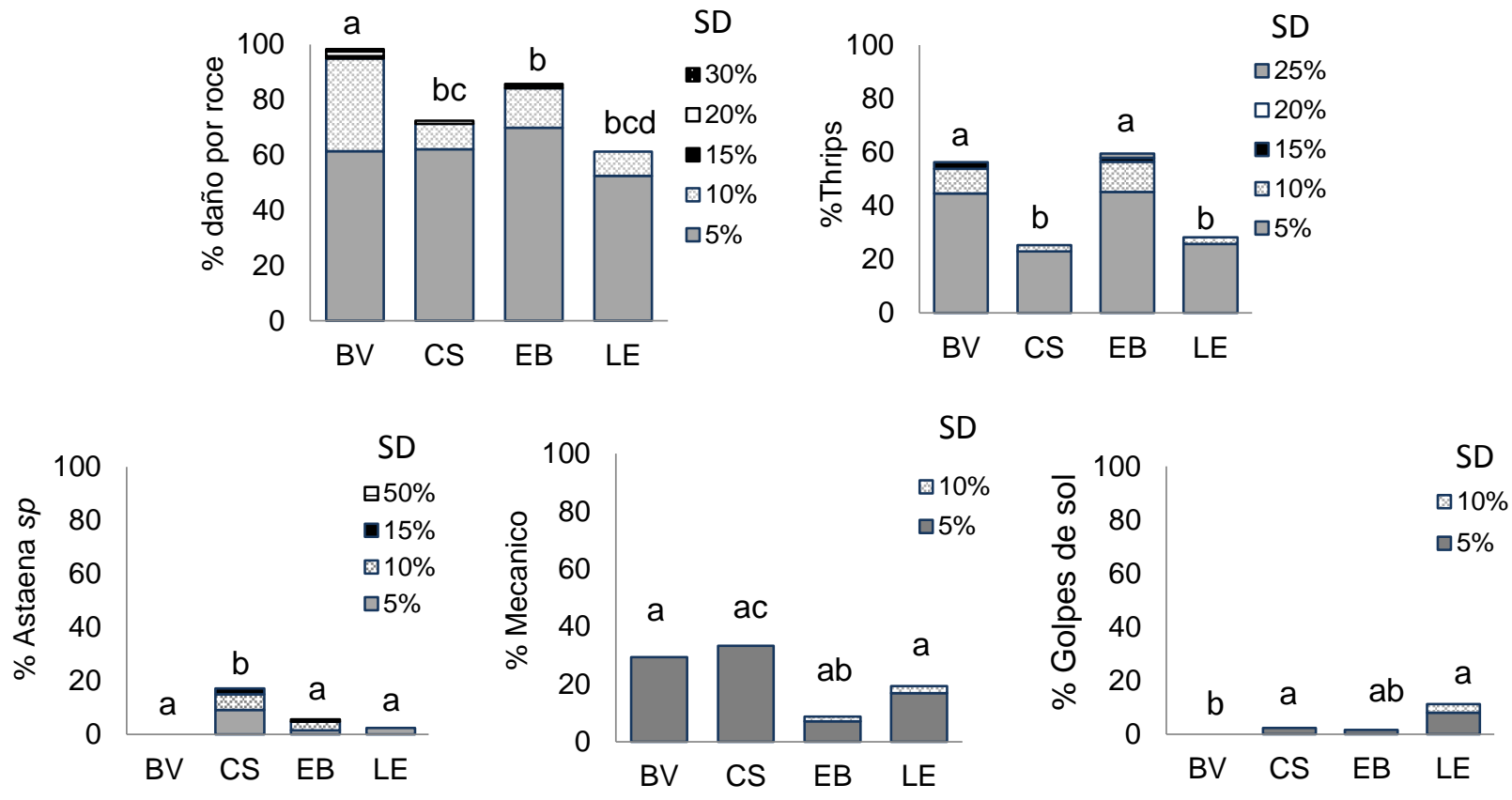

Fig. 2: Defectos externos después de la cosecha vs Fincas BV, EB, CS, LE y severidad de daño (SD) (5 al 50\%), 540 frutos en total evaluados $n=135$ por finca, Promedios con letras distintas indican diferencia estadísticamente significativa $(P \leq 0,05)$

El peso es un parámetro de selección que tiene influencia en la intención de compra de los consumidores de acuerdo al país de comercialización, Este peso después de la cosecha, es agrupado por determinados calibres definidos por la normatividad del país de destino (NMX, 2006; 1994; NTE, 2009; OECD, 2004). En este estudio las fincas que tuvieron los mayores pesos en el momento de cosecha fueron LE (124-380g) y EB (184 - 322g), seguidas de BV (168-286g) y CS (98-296g). Hubo diferencias de peso entre fincas a pesar de tener el mismo IM (Figura 3); esto puede ser debido al clima (temperatura y humedad relativa) y altura sobre el nivel del mar (Bernal et al., 2014; Salazar et al., 2016); sin embargo, se observa que la finca EB (2464msnm) y CS $(2411 \mathrm{msnm})$ tienen alturas y climas similares, pero los calibres de cosecha tuvieron diferencias muy marcadas en cuanto a peso. Otros autores se inclinan más por las condiciones de manejo del cultivo (fertilizaciones, podas, riego) los cuales tienen impacto en el crecimiento vegetativo y características nutricionales (Arpaia et al., 2004; Hofman et al., 2002).

En la figura 3, el IM de 30\%, tuvo diferencias estadísticamente significativas $(\mathrm{P}<0,05)$ comparado con los IM22 y $26 \%$, Esto es debido a un mayor tiempo de desarrollo del fruto, ya que durante esa etapa, el fruto de aguacate crece a medida que trascurre el tiempo, aumentando contenido de solidos no grasos y aceite. Para el IM 22 y $26 \%$, en las fincas CS y BV no se encontraron diferencias estadísticamente significativas y para EB y LE sí se encontraron diferencias, aunque con tendencias diferentes; es decir, que no siempre a mayor IM existe un mayor peso en los frutos. Los resultados obtenidos por Arpaia et al., (2015) son similares, a medida que aumentó el contenido de materia seca o IM, no aumento el peso de los frutos; aunque los autores tampoco obtuvieron diferencias significativas en peso de cosecha para IM superiores a $30 \%$, diferente a lo hallado en este estudio. La tabla 3 muestra la distribución de calibres por fincas exportadoras durante el periodo de cosecha. Los rangos de peso se encuentran entre 98 y $380 \mathrm{~g}$, cumpliendo con los parámetros de calidad establecido por la OECD, (2004) para aguacate Hass. Por su parte, los aguacates de preferencia en Europa, son los de calibre 16 a 20 (López y Barrera, 2008), siendo la finca EB la que tuvo mayor porcentaje de frutos dentro de este grupo $(69,1 \%)$, seguido de la finca BV con $55,9 \%$, La finca que menor porcentaje tuvo dentro de este grupo de calibres fue CS con $36,3 \%$. 

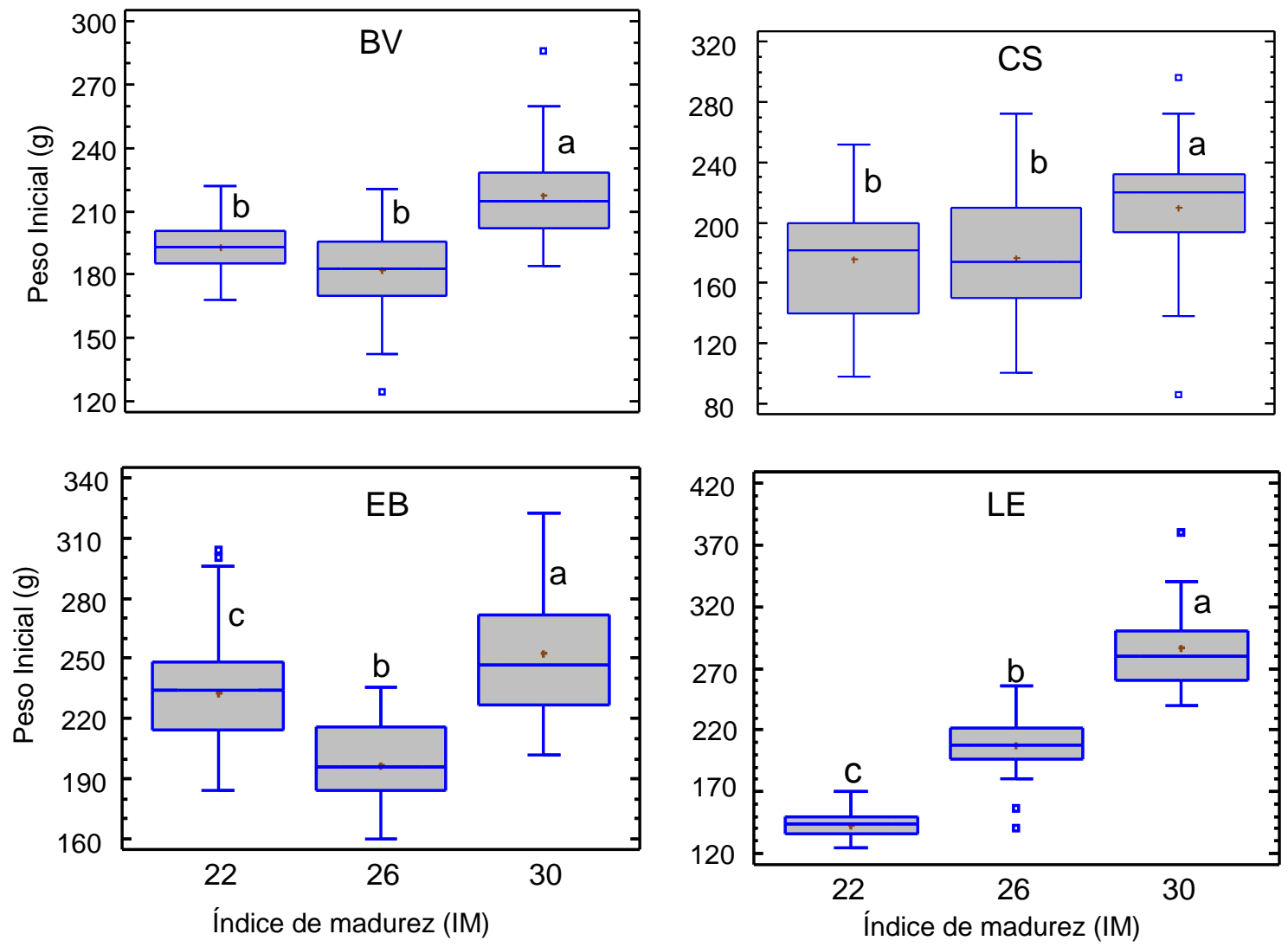

Fig. 3: Peso inicial después de la cosecha, Valores con letras distintas por finca indican diferencia estadísticamente significativa $(P \leq 0,05)$. Tukey HSD del 95\%

Tabla 3: Distribución de calibres encontrados por finca según la normatividad de la Unión Europea para frutos de aguacate

\begin{tabular}{|c|c|c|c|c|}
\hline $\begin{array}{c}\text { Distribución de calibres } \\
\text { en gramos }(g) \text { UE } \\
\text { (OECD, 2004) }\end{array}$ & $B V(\%)$ & $C S(\%)$ & $E B(\%)$ & $L E(\%)$ \\
\hline $10(366-460)$ & 0,0 & 0,0 & 0,0 & 1,6 \\
\hline $12(306-365)$ & 0,0 & 0,0 & 0,8 & 6,5 \\
\hline $14(266-305)$ & 0,8 & 3,4 & 12,7 & 15,3 \\
\hline $16(236-265)$ & 4,2 & 10,3 & 24,6 & 15,3 \\
\hline $18(211-235)$ & 15,3 & 20,7 & 27,8 & 9,7 \\
\hline $20(191-210)$ & 36,4 & 16,1 & 16,7 & 11,3 \\
\hline $22(171-190)$ & 41,5 & 24,1 & 17,5 & 4,8 \\
\hline $24(<156)$ & 1,7 & 25,3 & 0,0 & 35,5 \\
\hline
\end{tabular}

El croma $a^{*}$ presentó valores entre -11 y -12 para las fincas BV, EB y LE (Figura 4); para el croma b* los resultados están en el rango de 17 y 19 y la luminosidad $\left(L^{*}\right)$ entre 37 y 38 , estos valores en las coordenadas $L^{*}, a^{*}$ y $b^{*}$ corresponden a un color verde con tendencia a tonalidades oscuras u opacas. La finca CS tuvo un valor de $a^{*}$ menor a las demás fincas $(-14,24 \pm 1,5)$, mayor para el croma $b^{*}(22,8 \pm 2,5)$ y la luminosidad $(41,04 \pm 2,5)$. Estos valores se encuentran dentro de los rangos reportados para aguacate Hass en Antioquia y España en frutos entre 0 y 3 días de cosecha (Márquez et al., 2014; Pérez de los Cobos, 2013; Rodríguez y Henao, 2016). No se observó efecto significativo sobre las coordenadas $L^{*} a^{*} b^{*}$ por IM en las 4 fincas, esto indica que estas variables por si solas, no permiten identificar el índice de madurez de cosecha. Sin embargo, en la figura 5 se observa que el índice $\mathrm{h}^{\circ}$, permite agrupar los frutos por IM y tuvo un efecto estadísticamente significativo $(P<0,05)$ para 3 de las 4 fincas evaluadas $(B V, E B$ y $L E)$. Estos resultados fueron similares a los encontrados por Pérez de los Cobos (2013), donde no se encontraron diferencias de color CIE $L^{*} a^{*} b^{*}$, durante el desarrollo de los frutos, pero en la coordenada polar $\mathrm{h}^{\circ}$, se encontró un descenso del valor durante el crecimiento de la fruta. Por tanto, el ángulo de tono puede ser útil en la selección de fruta de manera no destructiva, sin tener que recurrir a metodologías de análisis como materia seca o determinación del contenido de aceite. 

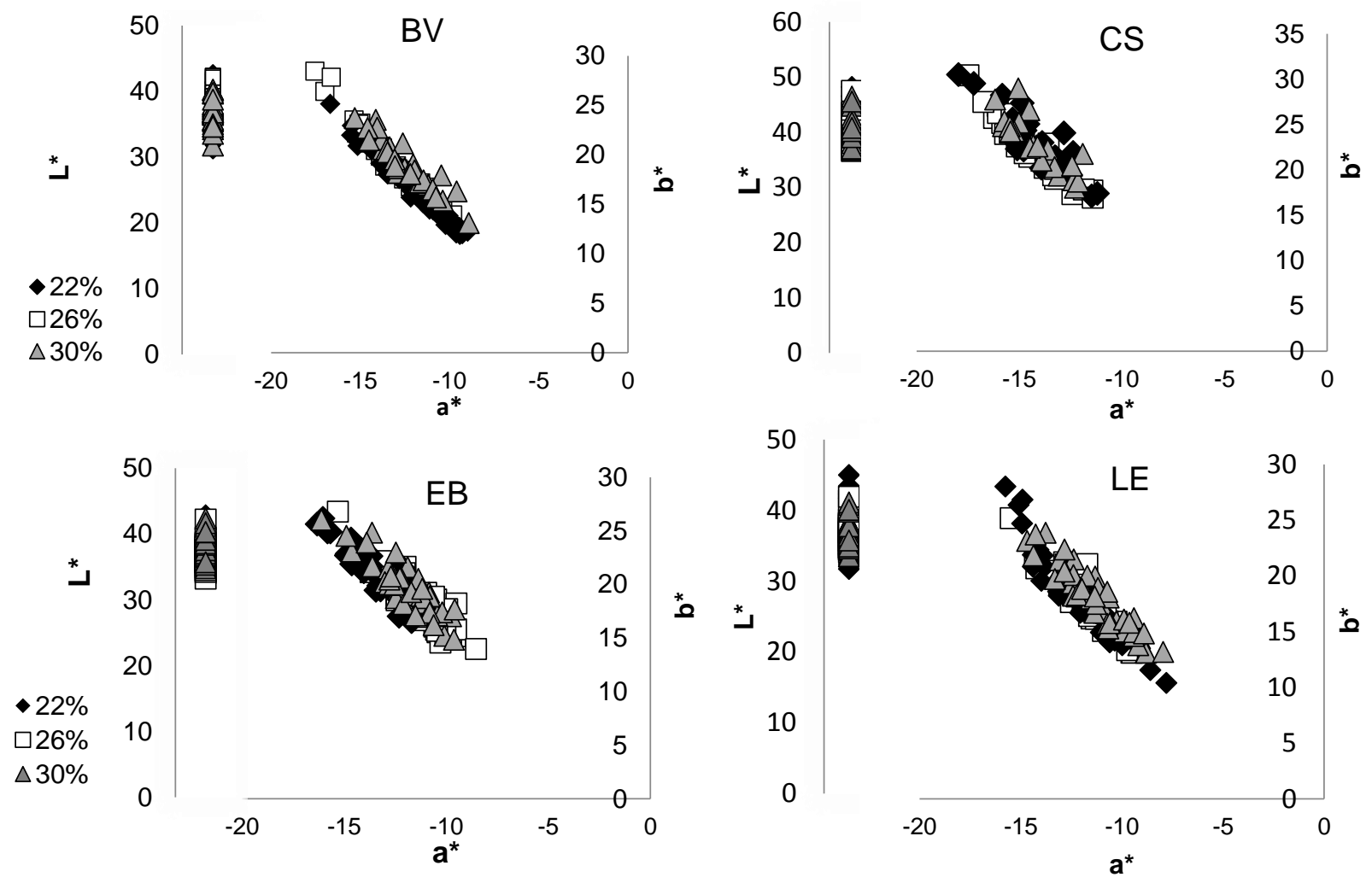

Fig. 4: Efecto del Índice de madurez (IM) 22, 26 y 30\% sobre el color (CIE L*a*b*) del epicarpio de frutos de aguacate 'Hass' después de la cosecha, en 4 fincas seleccionadas
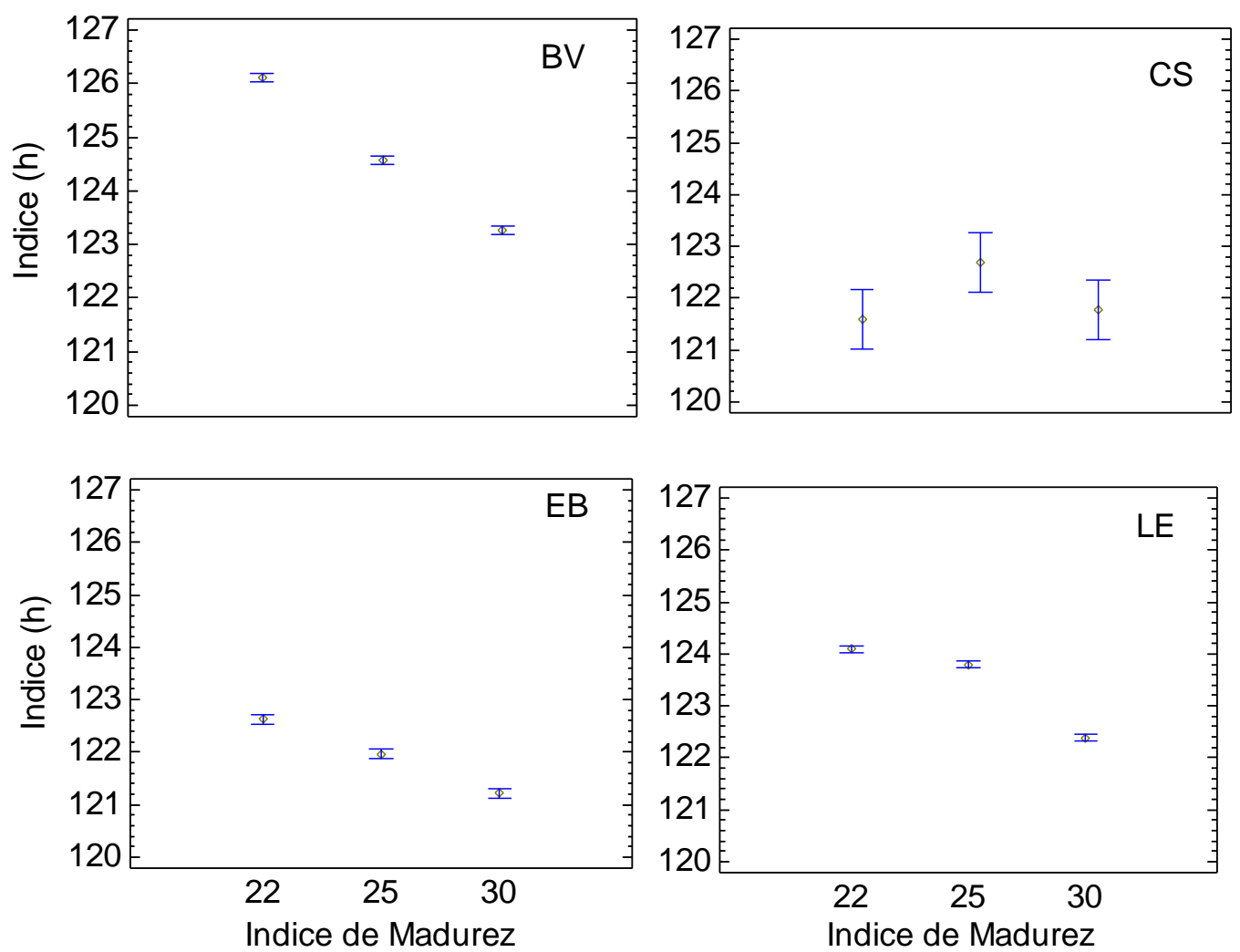

Fig. 5: Efecto del índice de madurez sobre el índice ho (ángulo de tono) $\left(0-360^{\circ}\right)$ de frutos de aguacate 'Hass', Prueba de rangos múltiples de Tukey, $\operatorname{HSD}(\mathrm{P} \leq 0,05)$ 


\section{Parámetros de calidad medidos después del almacenamiento}

Los daños por frio son un criterio de inspección y control de calidad de los frutos de aguacate después del almacenamiento. Se distinguen como manchas oscuras en la piel y pueden favorecer daños en la pulpa antes de que el fruto sea madurado y cortado (Ferreyra y Defilippi, 2012; Ochoa, 2014).

Las fincas EB y BV no presentaron daños por frio superiores al $1 \%$ y CS no fue mayor del $5 \%$ de los daños; el IM y TA no tuvieron efecto significativo para estas tres fincas a la temperatura de conservación $\left(5^{\circ} \mathrm{C}\right)$ (Figura 6). Estos resultados son similares a los reportado por Dixon et al., (2003) para almacenamiento a $5^{\circ} \mathrm{C}$ a diferentes momentos de cosecha, Para los tratamientos ms304, ms264, y ms265 de la finca LE, se encontraron daños por frío significativos $(P<0,05)$ por encima de 5,10 y $20 \%$ respectivamente. Esto podría afectar calidad de la pulpa de los frutos. Estos daños pueden deberse a la poca resistencia de los frutos al estrés por frío durante almacenamiento prolongado (4 o 5 semanas), y puede estar relacionado con deficiencias en nutrientes (minerales) de la pulpa (Ferreyra y Defilippi, 2012).

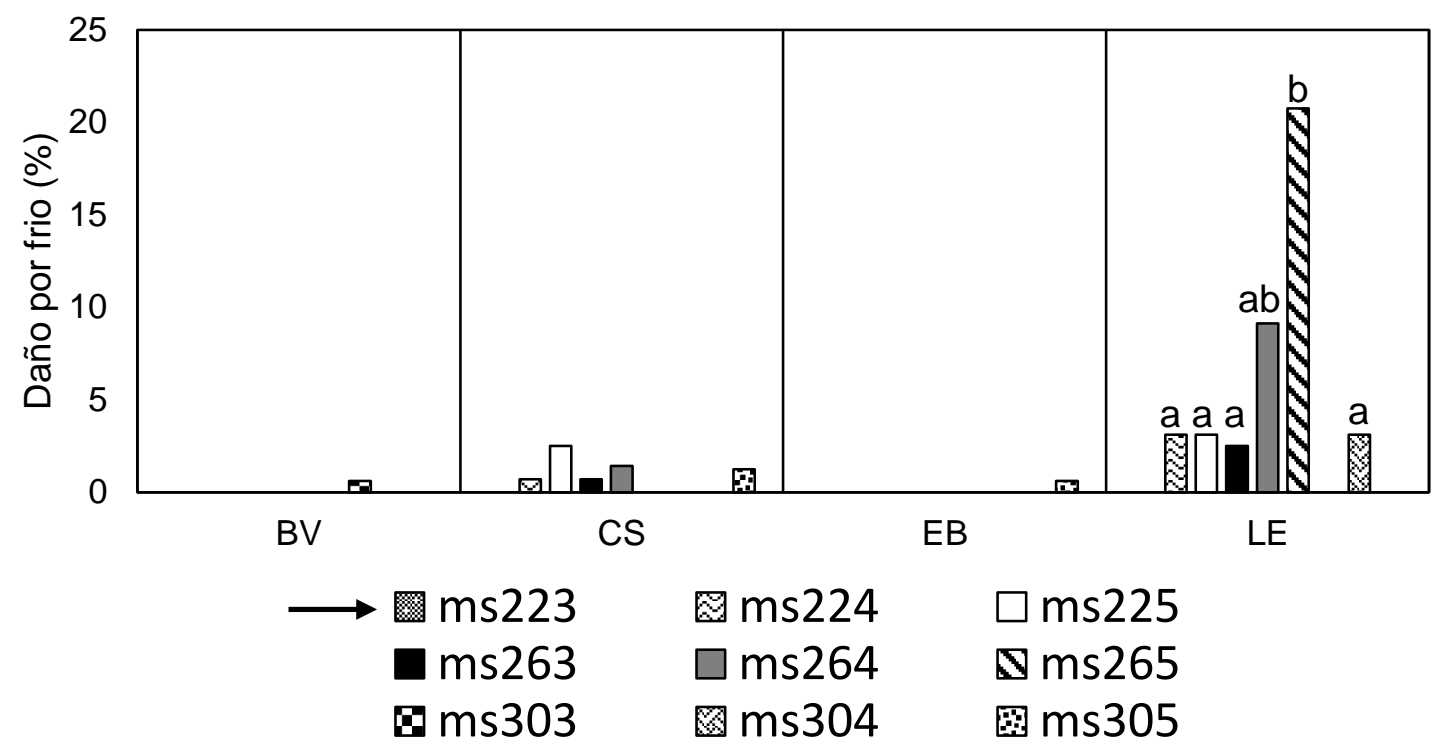

Fig. 6: Porcentaje de daño por frío en aguacate 'Hass' por tratamiento IM= índice de madurez ms22, ms26, ms30 y Tiempo de almacenamiento en frío (3, 4, 5 semanas), Finca LE con letras indica diferencias estadísticamente significativas entre tratamiento Tukey HSD $(\mathrm{P} \leq 0,05)$.

La pérdida de peso y deshidratación durante el almacenamiento y la maduración, determinan la vida útil y calidad del aguacate maduro. En la tabla 4 se observa un efecto significativo $(P<0,05)$ de TA sobre la pérdida de peso del aguacate en refrigeración y maduración (PA y PM) para todas las fincas. Por lo tanto, como se esperaba, a medida que incrementa las semanas de almacenamiento aumenta la pérdida de peso, sobre todo en la $4^{a}$ y $5^{a}$ semana, debido según la literatura a la transpiración y actividad metabólica de los frutos en estas dos etapas. En investigaciones realizadas en Sudáfrica a 4 semanas de almacenamiento, los frutos perdieron cerca del $8 \%$ de peso $\left(5,5^{\circ} \mathrm{C}\right.$ HR $\left.90 \%\right)$ (Blakey et al., 2014), siendo el valor de PA muy inferior en este estudio hasta la $5^{\text {a }}$ semana.

En los frutos que fueron llevados directamente a maduración (semana 0), fue similar el comportamiento de PM con respecto a la semana 3 y 4 de almacenamiento; con esto se podría decir que a 3 y 4 semanas (21 y 28 días) y maduración (aprox, 7 días) los frutos no han sufrido estrés por frío traducidas en pérdida de peso. Resultados similares fueron encontrados para frutos cultivados en España donde la pérdida de peso acumulada o PM no superó el 6,5\% sin refrigeración (Pérez de los Cobos, 2013), También, considerando la alta correlación existente entre la respiración de los frutos y la pérdida de peso, se puede decir que no existieron tasas de respiración que afectaran la calidad del fruto hasta la 4 a semana de almacenamiento en frio (Blakey et al., 2014).

En cuanto al IM, se observaron diferencias significativas $(P<0,05)$ sobre PA y PM, para las fincas BV y LE; en CS y EB no se hallaron diferencias. Por consiguiente, el índice de madurez no fue determinante en la pérdida de peso de los frutos, a diferencia del tiempo de almacenamiento. 
Tabla 4: Efecto del índice de madurez (IM) y el tiempo de almacenamiento (TA) sobre él valor promedio de la pérdida de peso después del almacenamiento $(P A)$ y maduración $(P M)$ del aguacate 'Hass'. Prueba de Tukey, $(P<0,05)$.

\begin{tabular}{|c|c|c|c|c|c|c|c|c|}
\hline$I M$ & \multicolumn{2}{|c|}{$B V$} & \multicolumn{2}{|c|}{$C S$} & \multicolumn{2}{|c|}{$E B$} & \multicolumn{2}{|c|}{$L E$} \\
\hline & $P A$ & PM & PA & PM & PA & PM & PA & PM \\
\hline $22 \%$ & $3,268 \mathrm{a}$ & $5,466 a$ & $5,315 b$ & $7,390 \mathrm{a}$ & $5,244 b$ & $8,236 a$ & $6,658 \mathrm{c}$ & $8,647 b$ \\
\hline $26 \%$ & $3,852 \mathrm{a}$ & $5,892 a b$ & $4,936 \mathrm{~b}$ & $6,771 a b$ & $5,204 \mathrm{~b}$ & $7,577 \mathrm{a}$ & $3,797 \mathrm{a}$ & $6,696 \mathrm{a}$ \\
\hline $30 \%$ & $5,408 b$ & $6,396 \mathrm{~b}$ & $4,495 \mathrm{~b}$ & $6,288 \mathrm{~b}$ & $4,576 \mathrm{~b}$ & $7,440 \mathrm{a}$ & $5,728 b$ & $8,133 a b$ \\
\hline TA & & & & & & & & \\
\hline 0 & & $5,163 \mathrm{a}$ & & $6,060 \mathrm{a}$ & & $6,876 \mathrm{a}$ & & $6,837 \mathrm{a}$ \\
\hline 3 & $2,715 a$ & $5,315 a$ & $3,964 \mathrm{a}$ & $5,888 \mathrm{a}$ & $4,207 \mathrm{a}$ & $7,399 a b$ & $4,495 \mathrm{a}$ & $7,442 \mathrm{a}$ \\
\hline 4 & $4,662 \mathrm{~b}$ & $5,840 \mathrm{a}$ & $4,659 \mathrm{a}$ & $6,969 \mathrm{a}$ & $4,639 a$ & $8,264 \mathrm{~b}$ & $5,792 \mathrm{~b}$ & $8,255 \mathrm{a}$ \\
\hline 5 & $5,152 b$ & $7,355 \mathrm{~b}$ & $6,123 b$ & $8,351 \mathrm{~b}$ & $6,179 \mathrm{~b}$ & $8,467 \mathrm{~b}$ & $5,896 \mathrm{~b}$ & $8,767 \mathrm{a}$ \\
\hline \multicolumn{9}{|c|}{ Análisis de varianza ( $p$-valor) } \\
\hline $\mathrm{IM}$ & 0,000 & 0,011 & 0,069 & 0,014 & 0,049 & 0,172 & 0,000 & 0,041 \\
\hline TA & 0,000 & 0,000 & 0,000 & 0,000 & 0,000 & 0,008 & 0,0002 & 0,152 \\
\hline IM X TA & 0,063 & 0,0002 & 0,132 & 0,232 & 0,417 & 0,041 & 0,795 & 0,818 \\
\hline
\end{tabular}

\section{Parámetros de calidad medidos en los frutos de aguacate en madurez de consumo}

En la tabla 5 se muestra el efecto del IM y la del TA sobre el color en la cáscara de los frutos en madurez de consumo. En este cuadro se pueden observar diferencias estadísticamente significativas $(p<0,05)$ en la mayoría de las coordenadas de color, tanto para IM, como TA en las fincas EB, BV y LE. La variabilidad del color por finca están ligadas a las zonas de cultivo y condiciones pre cosecha, indicando que es importante en planta postcosecha agrupar por IM y características de cultivo similares, para disminuir la heterogeneidad del color durante la maduración de los frutos (Fuentealba et al., 2017). La coordenada de croma a* mostró valores positivos bajos $(0,43$ a 7,0$)$ para todas las fincas, esto corresponde a un color rojo tenue o púrpura en todos los frutos, Según, Cox et al., (2004), el ambiente en el cual los frutos crecen y son cultivados, ocasiona que en la maduración pase a una coloración purpura o directamente negra en la cascara del aguacate a madurez de consumo. El aguacate de Colombia bajo las condiciones de cultivo estudiadas tiende a coloraciones púrpuras similares a los aguacates de Chile o Nueva Zelanda y difiere al producido en california (USA), donde cambia a negro (Cox et al., 2004; Ferreyra y Defilippi, 2012).

También, se observó que al aumentar el IM de cosecha para las fincas, el valor de a* disminuyó, presentando una influencia clara del IM sobre el color del epicarpio de los frutos. Este comportamiento pudo estar debido a una mayor síntesis de antocianinas (3-O-glucósido cianidina) en los frutos con IM mayor, lo que puede estar ligado al contenido de clorofila de la cáscara al momento de la cosecha (Cox et al., 2004). Los daños patológicos, desórdenes fisiológicos (daños externos o internos) y la heterogeneidad en la maduración son aspectos importantes de la calidad postcosecha de los frutos (Ferreyra y Defilippi, 2012). En la figura 7 se presentan los gráficos de superficie de respuesta del logaritmo del área afectada (AA) en función del TA e IM para cada finca. En esta figura se observa un claro aumento del AA (oscurecimiento de pulpa) a mayores tiempos de almacenamiento $\left(5^{\mathrm{a}}\right.$ semana $/ 35$ días a $\left.5^{\circ} \mathrm{C}\right)$ para todas las fincas. El frío puede causar lesiones que afectan la integridad de las membranas celulares, lo cual favorece la liberación de enzimas y sustratos durante el almacenamiento prolongado, provocando que la peroxidasa y polifenol oxidasa reaccionen con los sustratos fenólicos y se observen las coloraciones marrones en el mesocarpio del fruto (Ferreyra y Defilippi, 2012; Hershkovitz et al., 2005; Zauberman y Jobin, 1995).

A pesar de esto, a la $3^{a}$ semana de almacenamiento en frío, en las 4 fincas, se encontró que para un índice de madurez de $26 \%$, el AA estuvo localizada por debajo del $1 \%$, sin utilizar ningún tratamiento diferente a la refrigeración. Otras Investigaciones han logrado similares resultados combinando la acción de inhibidores de madurez (1-Metilciclopropeno) con el almacenamiento en frío (Hershkovitz et al., 2005; Zauberman y Jobin, 1995). Esto sugiere que se debe controlar el almacenamiento del aguacate y los IM de cosechas; es decir, que si se tienen aguacates con IM de 22 o 30\% se deben almacenar bajo refrigeración en un tiempo no muy prolongado para que a nivel celular no ocurra ruptura de membranas, lo cual promovería la presencia de daños de la pulpa.

La finca EB tuvo el valor más bajo AA en comparación con LE, BV y CS (figura 7). Esto puede estar ligado a las condiciones pre-cosecha como nutrición mineral, condiciones hídricas y prácticas de manejo de cada zona de cultivo, las cuales también juegan un papel importante sobre la calidad de frutos al final de la cadena (Ferreyra y Defilippi, 2012). 


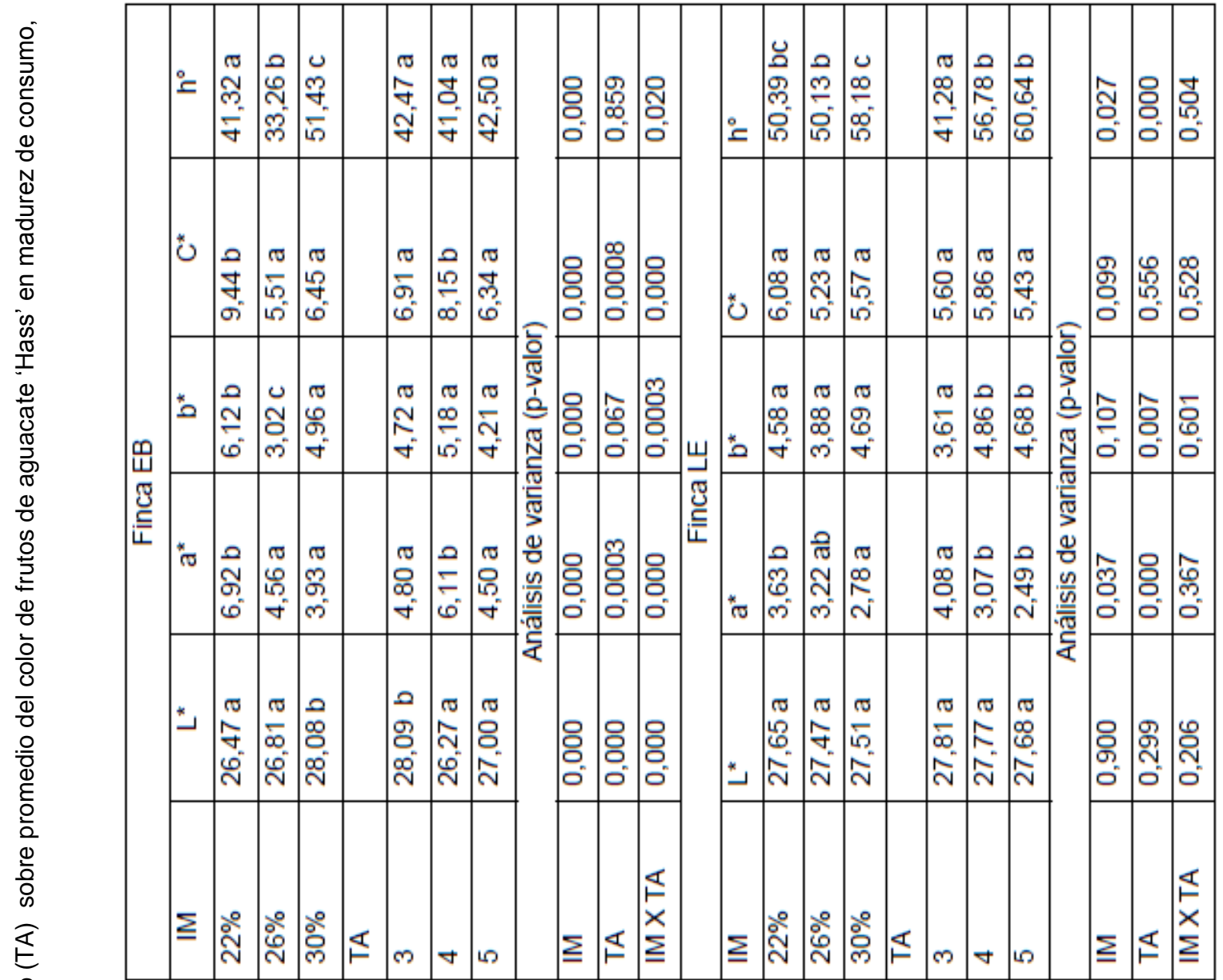

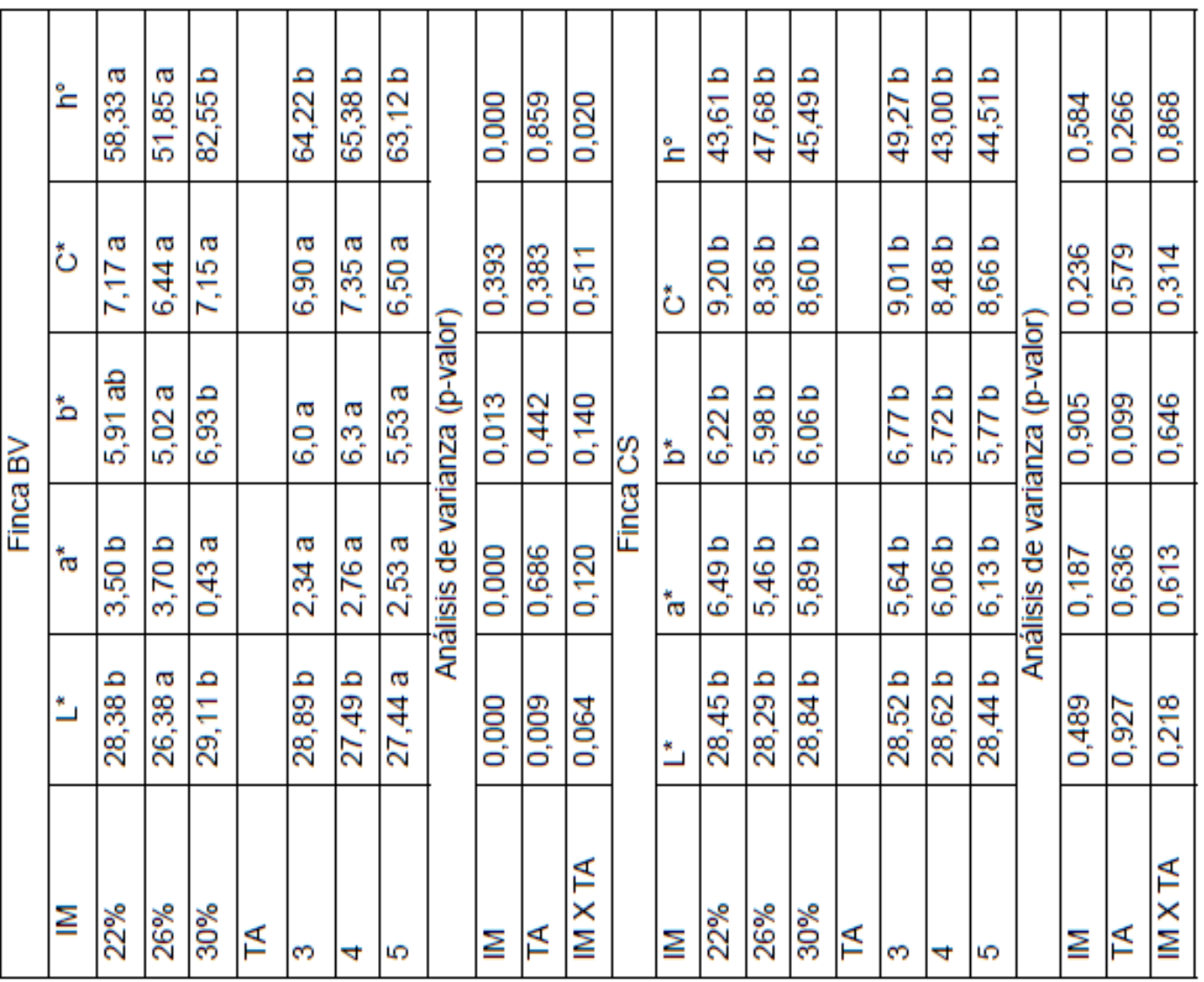



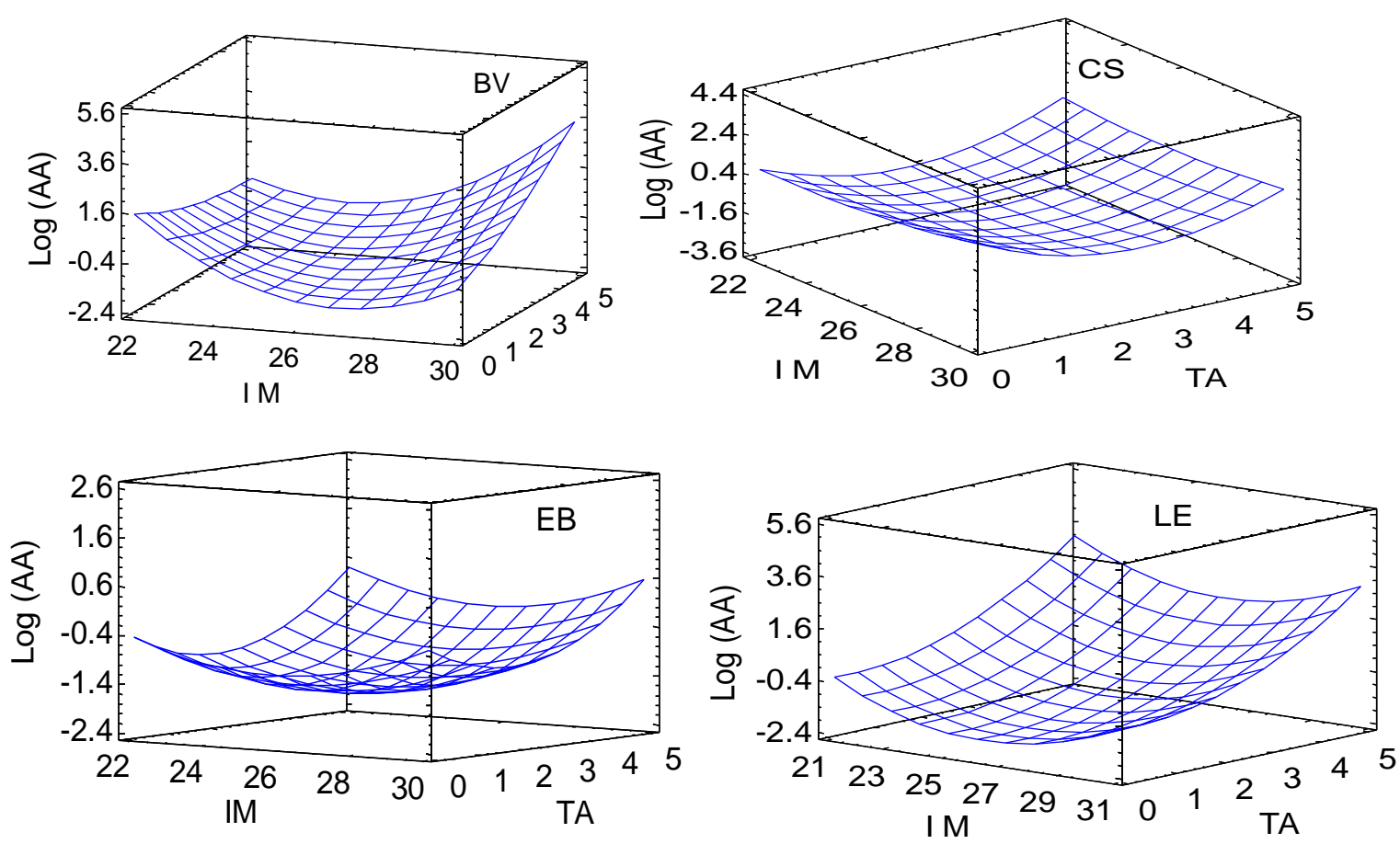

Fig. 7: Superficie de respuesta para AA (logaritmo de área afectada), Finca= BV, CS, EB, LE $(\mathrm{TA}=3,4$ y 5 semanas e $\mathrm{IM}=22,26$ y $30 \%)$,

\section{CONCLUSIONES}

En este estudio, los frutos de aguacate Hass se clasificaron en categoría extra y I, durante la recepción. EI peso de los frutos de aguacate depende de las condiciones de cultivo o variaciones ambientales de cada finca a pesar de tener los mismos índices de madurez. El índice $h^{\circ}$ (hue) o ángulo de tono podría servir como indicador de cosecha para clasificar los frutos. El índice de madurez y tiempo de almacenamiento en frío afectan los parámetros de calidad del aguacate Hass: Si se controlan el IM en las fincas y se realiza un almacenamiento en frío adecuado, se puede mejorar y controlar la calidad postcosecha de los frutos (pérdidas de peso altas, daños por frío y daños en pulpa). El área afectada de la pulpa por daños (parámetro de calidad determinante para el consumidor final), es menor cuando el aguacate Hass se cosecha a $26 \pm 2 \%$ de materia seca. Si se tienen frutos con IM de 22 o $30 \%$ se debe disminuir el tiempo de almacenamiento en frío para evitar daños en pulpa severos.

\section{AGRADECIMIENTOS}

A la Corporación de Investigación Agropecuaria (AGROSAVIA), por la financiación del proyecto por medio del Sistema General de Regalías Antioquia, Convenio Especial de Cooperación para la Investigación No, 4600001078, suscrito entre el Departamento de Antioquia, Secretaría de Agricultura, Universidad Nacional de Colombia Sede Medellín, Acta de Adhesión No, 2 "Índices cosecha y poscosecha para aguacate cv, Hass en Antioquia: cumplimiento de normas y requisitos demandados por los mercados internacionales.

\section{REFERENCIAS}

Agronet, Estadísticas Agronet. Producción de aguacate, área sembrada, rendimiento por hectárea (2017)

AOAC, Association of Official Analytical Chemists, Official Methods of Analysis, AOAC INTERNATIONAL. 934.060 (2000)

Arpaia, M.L., S. Collin, J. Sievert y D. Obenland, Influence of cold storage prior to and after ripening on quality factors and sensory attributes of "Hass" avocados, doi:doi.org/10.1016/j.postharvbio.2015.07.016, Postharvest Biology and Technology, 110, 149-157 (2015)

Arpaia, M. L., J. P. Van Rooyen, P. Hofman y A. Woolf, Grower practices will influence postharvest fruit quality, 2 Seminario Internacional de Paltos, 1-9. Quillota, Chile, 29 de septiembre - 1 de octubre (2004)

Bernal, J., C. Díaz y otros, Actualización Tecnológica y Buenas Prácticas Agrícolas (BPA) en el Cultivo de Aguacate. 2 $2^{\mathrm{a}}$ Ed., Libros publicados Corpoica, Antioquia, Colombia (2014)

Bill, M., D. Sivakumar, A.K. Thompson y L. Korsten, Avocado fruit quality management during the postharvest supply chain; doi: doi.org/10.1080/87559129.2014.907304, Food Review International, 30(3), 37-41 (2014) 
Blakey, R.J., S. Z. Tesfay, I. Bertling y J. P. Bower, Ripening physiology and quality of "Hass" avocado (Persea americana Mill.) after cold storage at $1^{\circ} \mathrm{C}$; doi: doi.org/10.1080/14620316.2014.11513134, Journal of Horticultural Science and Biotechnology, 89(6), 655-662 (2014)

Burdon, J., P. Connolly y otros cuatro autores, A meta-analysis using a logit non-linear mixed effects model for "Hass" avocado postharvest performance data, Postharvest Biology and Technology, 86, 134-140 (2013)

Cerdas, M., C. M. del Montero y O. Somarribas, Verificación del contenido de materia seca como indicador de cosecha para aguacate (persea americana) cultivar Hass en zona intermedia de producción de los santos, Costa Rica, Agronomía Costarricense, 38(1), 207-214 (2014)

Cox, K. A., T. K. McGhie, A. White y A. B. Woolf, Skin colour and pigment changes during ripening of "Hass" avocado fruit, doi: 10.1016/j.postharvbio.2003.09.008, Postharvest Biology and Technology, 31(3), 287-294 (2004)

Dixon, J., H.A. Pak y otros tres autores, New Zeland avocado fruit quality: the impact of storage temperature and maturity, doi:doi.org/10.1017/CBO9781107415324.004.NZ, Avocado Grower Association Annual Research Report, 3, 48-53 (2003)

Dreher, M.L. y A.J. Davenport, Hass avocado composition and potential health effects, doi: doi.org/10.1080/10408398.2011.556759, Critical Reviews in Food Science and Nutrition, 53(7), 738-50 (2013)

Ferreyra, R. y B. Defilippi, Factores de pre cosecha que afectan la postcosecha de la Palta Hass. Clima, Suelo y Manejo, La Cruz, Chile: CORFO, INIA, Ministerio de Agricultura de Chile (2012)

Fuentealba, C., R. Predrechi, I. Hérnandez y J. Saavedra, A statistical approach for assessing the heterogeneity of Hass avocados subjected to different postharvest abiotic stresses, doi: doi.org/10.4067/S0718-16202016000300002, Ciencia E Investigación Agraria, 43 (3), 356-365 (2017)

Hérnandez, I., C. Fuentealba, J.A. Oleata, B.G. Defilippi, R. Campos-vargas y R. Pedreschi. Factors associated with postharvest ripening heterogeneity of' Hass' avocados (Persea americana Mill) https://doi.org/10.1051/fruits/2016016 Review article of "Hass" avocados (Persea americana Mill). Fruits, 71(5), 259-268 (2015)

Hershkovitz, V., S.I. Saguy y E. Pesis, Postharvest application of 1-MCP to improve the quality of various avocado cultivars, doi: doi.org/10.1016/j.postharvbio.2005.05.003, Postharvest Biology and Technology, 37(3), 252-264 (2005)

Hofman, P.J., J. Bower y A. Woolf, Harvesting, packing, postharvest technology, transport and processing. In The avocado: botany, production and uses, Doi: doi.org/10.1079/9781845937010.0489 Wallingford: CABI, 489-540 (2002)

López, L.F., E. Barrera, Diagnóstico de mercados internacionales para productos agroindustriales potenciales en Bolívar: el caso de aguacate. Serie de Avances de Investigación. Edición: IICA Biblioteca Venezuela. ISSN: 2145-0749. 40pp. Cartagena de Indias, Colombia (2008)

Márquez, C.J., D.P. Yepes, L. Sánchez y J. Osorio, Cambios Fisicoquímicos del aguacate (Persea americana Mill cv "Hass" en postcosecha para dos municipios de Antioquia. Temas Agrarios, 19(1), 32-47 (2014)

NMX FF-016-SCFI. Productos alimenticios no industrializados para uso humano - fruta fresca - Aguacate (Persea americana Mill). Especificaciones. Dirección General de Normas, México (2006)

NTE 1755. Norma Técnica Ecuatoriana: Frutas frescas. Aguacate. Requisitos. Instituto Ecuatoriano De Normalización. Instituto Ecuatoriano de Normalización. Quito. Ecuador (2009)

Ochoa, S., Enfermedades y desordenes fisiológicos más comunes del fruto de aguacate en postcosecha. Guía ilustrada para Técnicos y Empacadores. (M. Gonzales, 1를 Ed.) APEAM, A.C. México (2014)

OECD. Organisation for Economic Co-Operation and Development Organisation De Cooperation Et De Développement Économiques, International Standardization of Fruit and Vegetables (Avocado). 1-130. Unión European (2004)

Pedreschi, R., P. Muñoz y otros cinco autores, Metabolomics analysis of postharvest ripening heterogeneity of "Hass" avocadoes, Postharvest Biology and Technology, doi: doi.org/10.1016/j.postharvbio.2014.01.024 92, 172-179 (2014)

Pérez de los Cobos, R., Crecimiento y maduración del fruto en aguacate (Persea americana Mill.) cv. Hass. Tesis de pregrado, Universidad de Almería, Hortifruticultura (2013)

Rodríguez, P. y J. Henao, Maduración del Aguacate (Persea Americana Mill cv. Hass) y calidad de los Frutos. doi: doi.org/10.15446/agron.colomb. Agronomía Colombiana, I (1), 914-917 (2016)

Salazar, S., R. Medina y A. Álvarez, Evaluación inicial de algunos aspectos de calidad del fruto de aguacate 'Hass' producido en tres regiones de México, Revista Mexicana de Ciencias Agrícolas, 7 (2), 277-289 (2016)

United-Nations Ffv-42 United Nations: Unece: Standard concerning the marketing and commercial quality control of Avocados I. New York. USA (2010)

White, A., A. Woolf, A. P. Hofman y M.L. Arpaia, The International Avocado Quality manual. Plant and Food Research, Auckland, Nueva Zelanda (2009)

Zapata, J.E., A. M. Restrepo-Suárez y L. Arias, Cinética de la Deshidratación Osmótica del Aguacate (Persea americana), y Optimización del Color por Medio de Superficies de Respuesta, doi.org/10.4067/S0718-07642016000400003, Información Tecnológica, 27(4), 17-32 (2016)

Zauberman, G. y M. I. Jobin, Avocado (Persea americana Mill) quality changes in response to low-temperature storage, Postharvest Biology and Technology, 5, 235-243 (1995) 